\title{
Ultrasono-Cardiotomographic Evaluation of Histological Changes in Myocardial Infarction
}

\author{
Hisaharu Hikichi, M.D., and Motonao TANAkA, M.D.
}

\section{Summary}

The echo patterns on ultrasono-cardiotomogram were evaluated in relation to the histological changes of the myocardium. The ultrasonocardiotomographic data were collected from 9 consecutive patients with transmural infarction (3 patients with antero-septal, 5 with postero-inferior, 1 with antero-lateral infarction) and also from 11 dogs with experimental myocardial infarction. Analyses of data obtained led to the following conclusion.

1) A decrement of the thickness and deformity of the wall echo: They were detected by a series of azimuthal angle and level ultrasonocardiotomograms which were obtained by changing the direction and level of the ultrasonic beam through the heart along the long axis of the left ventricle.

2) An increment of echo-intensity of infarcted area: The intensity of abnormal echo of the infarcted area, when evaluated by using the intensity of the echo of normal pericardium as a reference of $\mathrm{OdB}$, was +10 to $+15 \mathrm{~dB}$ more intense than that of the normal myocardium.

3) The study of the echo patterns in comparison with the histology of myocardium indicated that the echoes related to scar could be classified into 4 types: i) cord-like echo, ii) scattered large nodular echoes, iii) narrow band-like echo, and iv) scattered small nodular echoes.

Types i), ii), and iii) indicated the formation of a transmural scar. Thus a possibility was suggested that histological characteristics of myocardial tissue could be estimated by a non-invasive method.

\section{Additional Indexing Words :}

Echo-intensity Wall thickness Abnormal echo pattern Sensitivity gradient tomographic method Azimuthal and level ultrasonocardiotomograms

$7 \mathrm{HE}$ normal myocardial tissue is led into necrosis and is replaced with 1 fibrotic tissue after obstruction of the coronary arteries. The occurrence of fibrotic tissue results in the changes of thickness and shape of myocardial

From the Department of Medical Engineering and Cardiology, the Research Institute for Chest Diseases and Cancer, Tohoku University, Sendai, Japan.

Address for reprint: Hisaharu Hikichi M.D., the Research Institute for Chest Diseases and Cancer, Tohoku University, Seiryo-machi 4-1, Sendai 980, Japan.

Received for publication April 26, 1980.

Manuscript revised September 3, 1980. 
wall and also in changes of echo-intensity. The purpose of this study is the non-invasive evaluation of the histological changes of the infarcted area of the left ventricle.

\section{Material and Methods}

Ultrasono-cardiotomographic data were collected from 9 consecutive patients (the age ranging from 28 to 60 years) with transmural myocardial infarction, and also from 11 dogs in which experimental myocardial infarction had been produced. Antero-septal infarction in 3 cases, postero-inferior infarction in 5 cases and anterolateral infarction in 1 case were diagnosed on the basis of ECG findings.

In all the patients of this study, the width of $Q$ wave exceeded $0.04 \mathrm{sec}$. Also typical chest pain associated with infarction and the time course of enzyme activity were taken into account for the establishment of diagnosis. The group of experimental animals consisted of 3 dogs with antero-septal infarction, 4 dogs with posteroinferior infarction, and 4 dogs with postero-lateral infarction. Two apparatus were used throughout this experiment. One was the same as that used in our previous report. ${ }^{1,2)}$ The other had an electronic sector scan device.

The patients were examined in supine position with the transducer placed at the left fourth intercostal space lateral to the left sternal margin (the standard position). A thin $50 \times 30 \times 10 \mathrm{~cm}$ vinyl bag filled with warm degasified water was placed on the chest wall of a patient in supine position with olive oil as acoustic couplant. The ultrasonic beam traveled in the water through the chest wall to the heart and the heart was cross-sectioned by an ultrasonic beam. The transducer was placed as closely as possible to the chest surface. Ultrasono-cardiotomograms were obtained by changing the direction and the level of ultrasonic beam through the left ventricle. Long axis and short axis ultrasono-cardiotomograms were obtained by scanning along the long and short axes of the left ventricle respectively.

Since it was impossible to obtain specimens for histology in the clinical study, an experimental animal model was used for the study of the relationships between the histological changes of the myocardium and echo patterns.

Experimental myocardial infarction was produced by ligation of the coronary arteries. A ligation of the left anterior descending coronary artery produced an antero-septal myocardial infarction. As a rule, occlusion of the circumflex coronary artery produced postero-lateral infarction and that of the posterior descending coronary artery postero-inferior infarction. After 1 to 2 months, the chest was reopened and the heart was removed for ultrasono-cardiotomographic study and for histological study of the myocardium using Elastica Masson-stained specimens. Two different transducers made of a concave disc of barium titanate were utilized. One had a resonance frequency of $2.25 \mathrm{MHz}$ and a diameter of $30 \mathrm{~mm}$. The other had a resonance frequency of $3.5 \mathrm{MHz}$ and a diameter of $20 \mathrm{~mm}$.

For the evaluation of echo intensity, the sensitivity gradient tomographic method was used. In this method several shects of tomograms were obtained by changing only the gain of the receiving amplifier at steps of $5 \mathrm{~dB}$. The echo intensity of fibrotic tissue was measured with reference to the echo-intensity of the pericardial echo which was set at OdB. 


\section{RESUlts}

1) Morphological changes of the myocardium in relation to the characteristics of echo on ultrasono-cardiotomogram

Fibrosis in myocardium produced abnormal echoes in the area that was identified as such by ultrasono-cardiotomography. The size of the fibrotic tissue was delineated on azimuthal and level ultrasono-cardiotomograms obtained by changing the direction and level of ultrasonic beam through the heart along the long axis of the left ventricle.

A postero-inferior infarction of the human heart was diagnosed exactly by ultrasono-cardiotomograms obtained by a scan along the long axis of the left ventricle (long axis ultrasono-cardiotomogram) with a transducer placed in the standard position. The size of the infarcted area was determined by a series of azimuthal and level ultrasono-cardiotomograms (Fig. 1). Posteroinferior infarction was also diagnosed by short axis ultrasono-cardiotomography. The myocardial echo thus obtained of the postero-inferior wall of
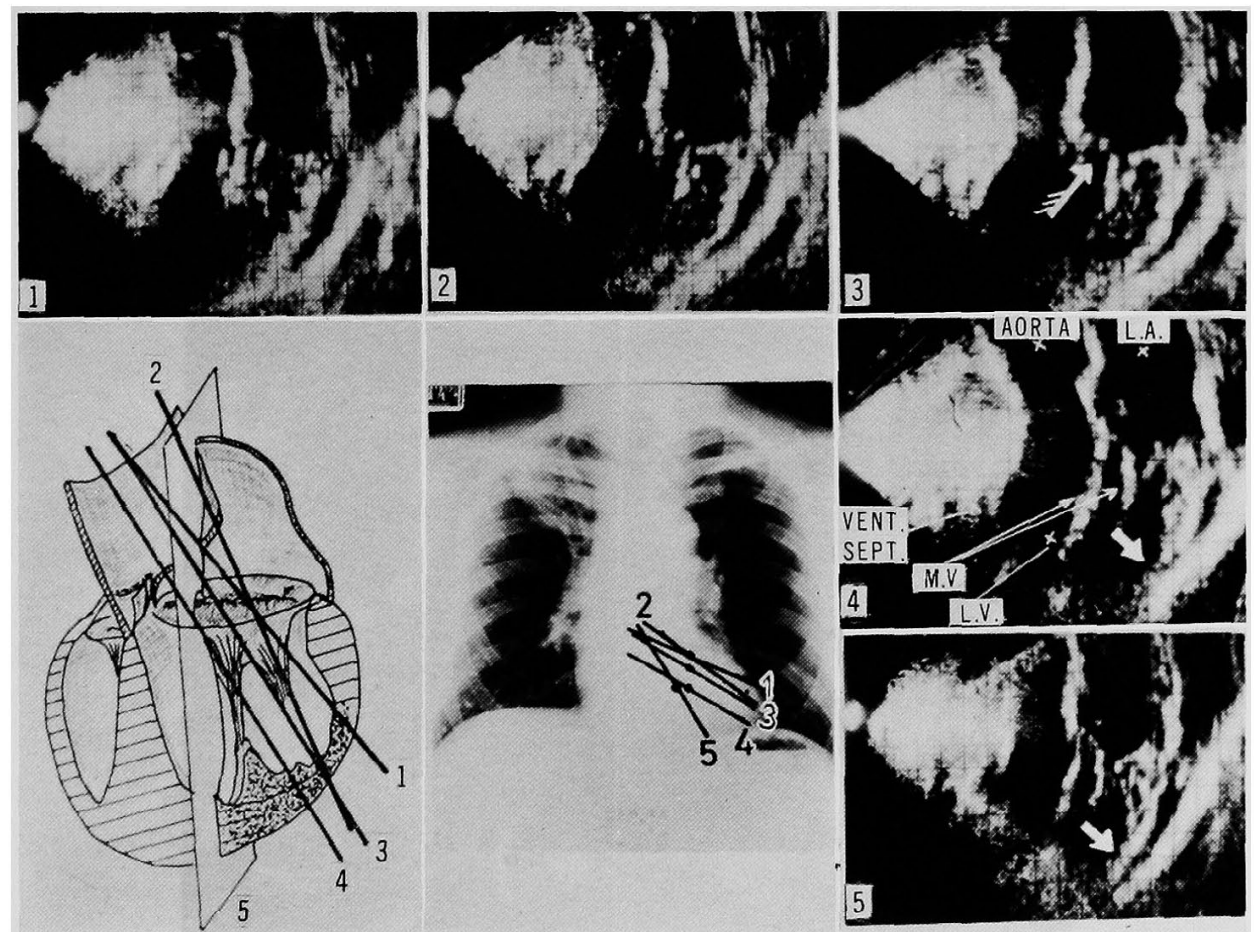

Fig. 1. Azimuthal and level ultrasono-cardiotomograms obtained by changing the direction and level of a cross-section of the heart along the long axis of the left ventricle with postero-inferior infarction. A decrement in thickness of myocardial echo in the infarcted area $(\rightarrow$ arrow) and mitral prolapse are demonstrated $(\hookrightarrow$ arrow). 
the left ventricle was thinner than that of healthy subjects (Fig. 2). A postero-inferior infarction of a dog could be diagnosed exactly by short-axis ultrasono-cardiotomography. Fig. 3 shows a narrow band-like echo of a scar

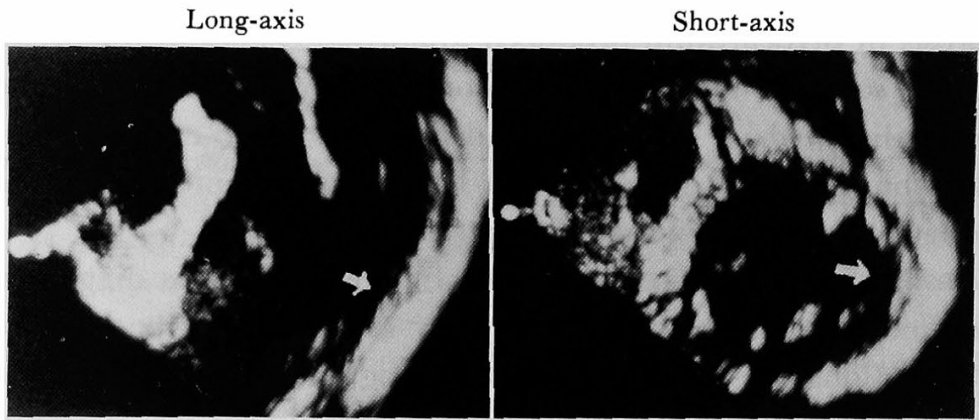

Fig. 2. Long and short axis ultrasono-cardiotomograms of human heart with postero-inferior infarction $(\rightarrow$ arrow). The thickness of myocardial wall echo is decreased.

Systole
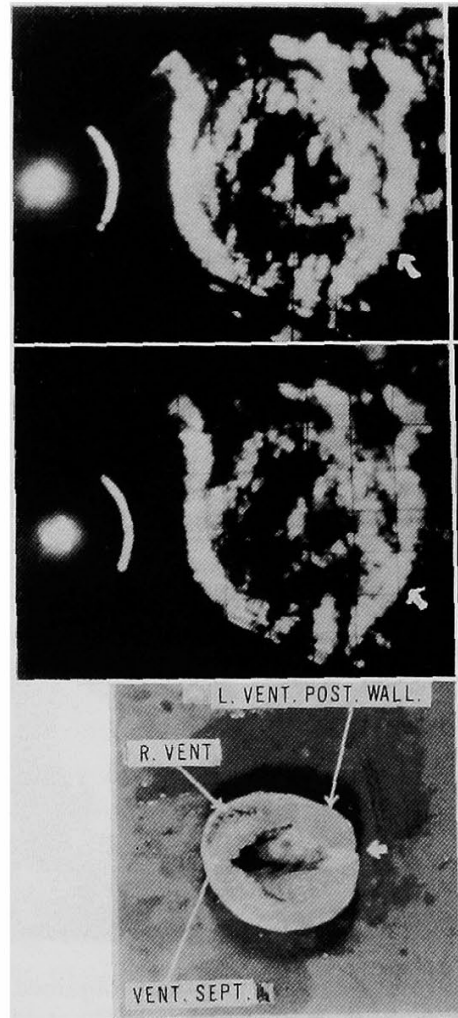

Fig. 3. Short axis ultrasono-cardiotomograms of an excised dog heart with postero-inferior infarction. A narrow band-like echo $(\rightarrow$ arrow) is observed. A postmorten specimen shown left below indicates that the echo patterns faithfully reflect the histological changes.

Diastole

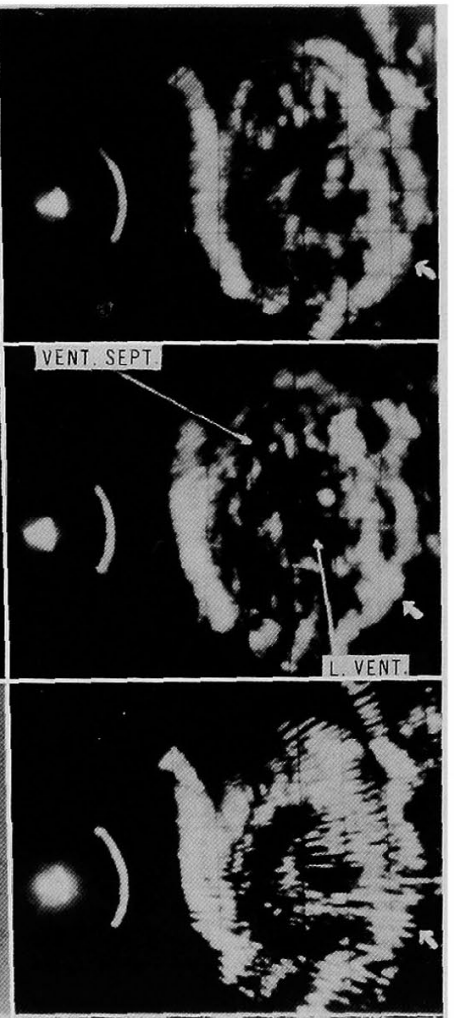


in myocardium. This ultrasono-cardiotomogram was obtained by a scan along the short axis.

Antero-septal infarction was diagnosed by a series of level ultrasonocardiotomograms obtained by changing the level of the cross-section of the heart along the long axis of the left ventricle with a transducer placed somewhat lower than usual. The echogram thus obtained showed that the apical portion of the septal wall and the anterior wall were thinner than those in healthy subject (Fig. 4).

In antero-septal infarction of a dog, a thin wall echo of myocardium was obtained by changing the azimuthal angle of the scanning planes from 1 to 6 (Fig. 5). Antero-lateral infarction was identified by a scan parallel to the ventricular septum with a transducer placed somewhat below and outside of the standard position (Fig. 6).

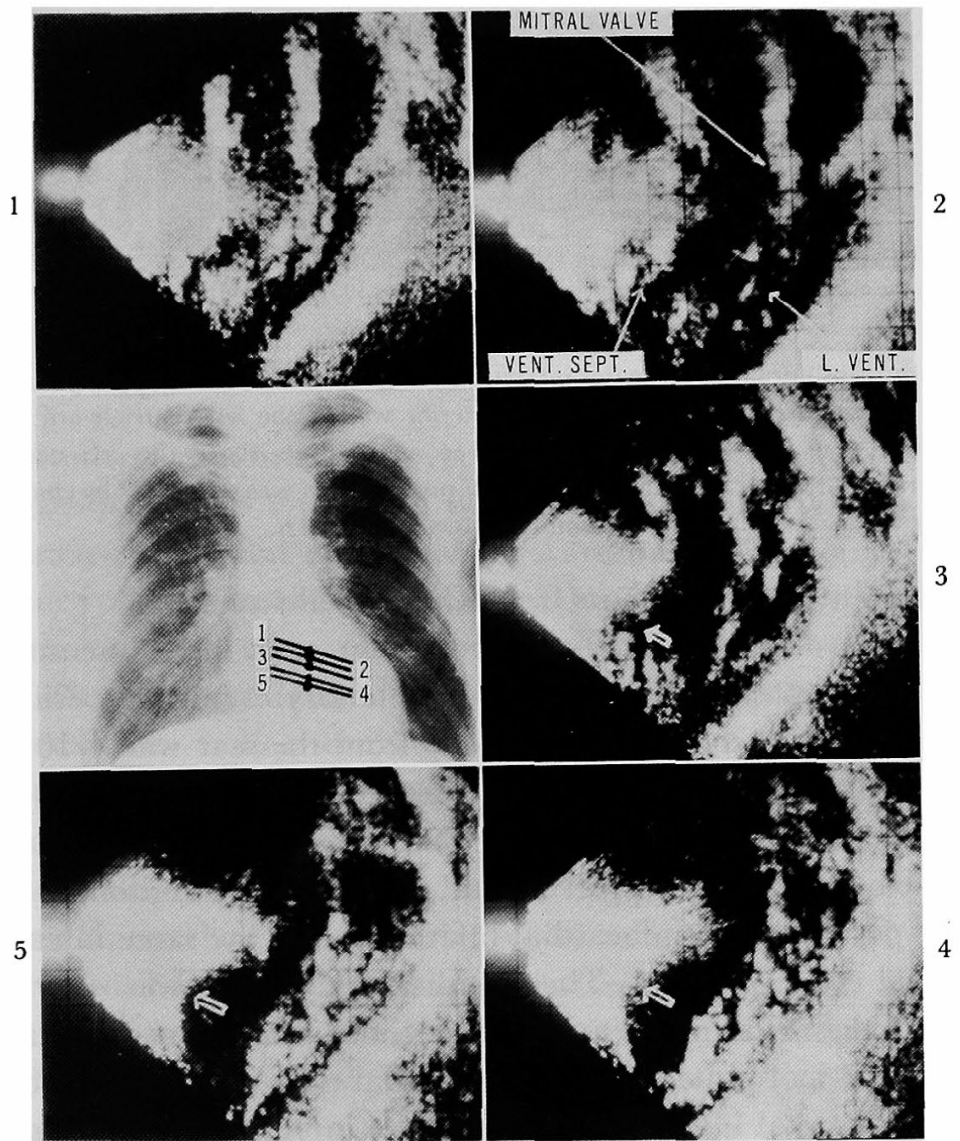

Fig. 4. Level ultrasono-cardiotomograms obtained by changing the level of a cross-section of the heart along the long axis of the left ventricle with antero-septal myocardial infarction. Both the apical portion of the septal wall and the anterior wall in this case are thinner than in normal heart, 
1

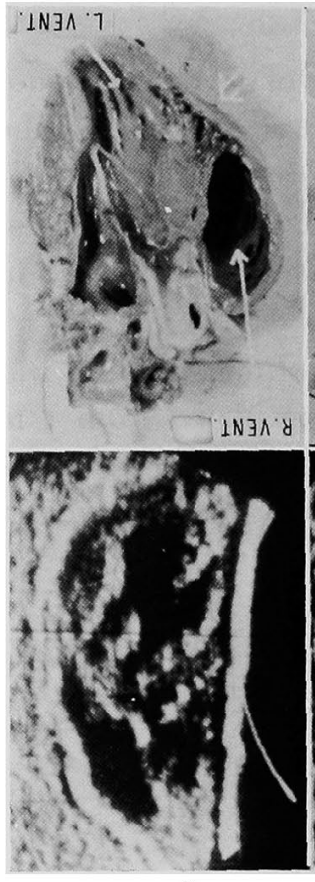

5
2
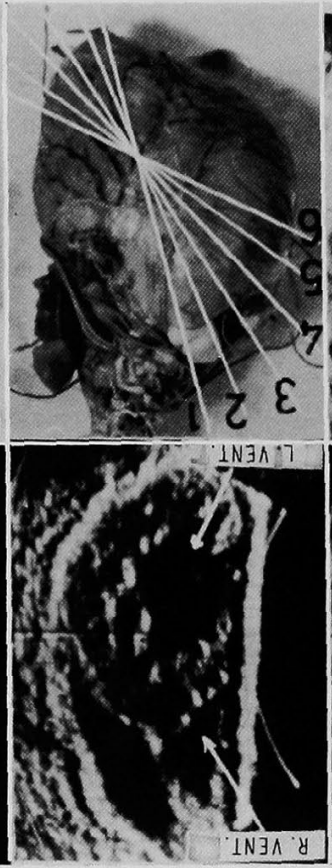

6
3
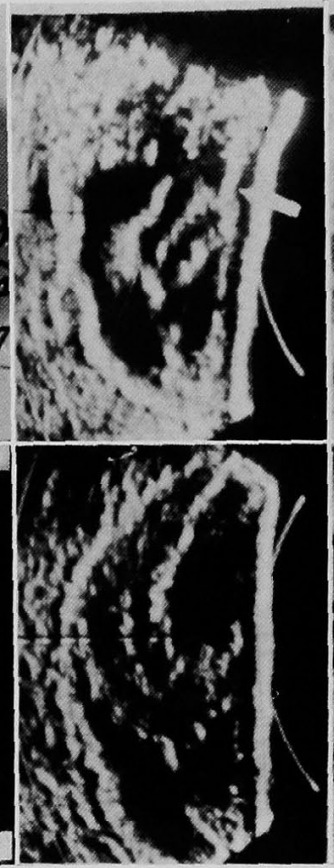

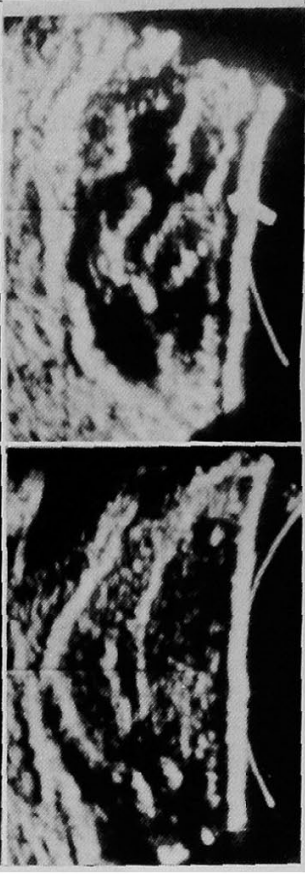

Fig. 5. Azimuthal ultrasono-cardiotomograms of an excised dog heart with transmural antero-septal infarction. The thickness of the apical portion of the septal wall and that of the anterior wall of the left ventricle are decreased. A cord-like echo ( $\rightarrow$ arrow) in myocardium shows the presence of a transmural scar. A thin wall echo of myocardium was diagnosed by changing the azimuthal angle of scanning planes from 1 to 6 .

2) The intensity of echoes from myocardial scar tissue

The echoes from the scar in myocardium were more intense than those from the intact myocardium. When the intensity of normal pericardial echo was used as a reference of $0 \mathrm{~dB}$, the echo from the scar was +10 to $+15 \mathrm{~dB}$ more intense than that of normal myocardium (Fig. 7), because pericardial ccho was +20 to $+25 \mathrm{~dB}$ more intense than myocardial echo in human heart.

On the other hand, in experimental myocardial infarction, the echo from the area of posterior subendocardial infarction had the same intensity as that of pericardial echo (Fig. 8). The intensity of this echo was +10 to $+15 \mathrm{~dB}$ more intense than that of normal myocardium, because pericardial echo was +10 to $+15 \mathrm{~dB}$ more intense than myocardial echo in an excised dog heart.

In antero-septal infarction also the echo-intensity was increased and appeared at a level of $+10 \mathrm{~dB}$. This echo was +5 to $+10 \mathrm{~dB}$ more intense than that of normal septum (Fig. 9).

3) Correlation between the echo patterns and histological changes 


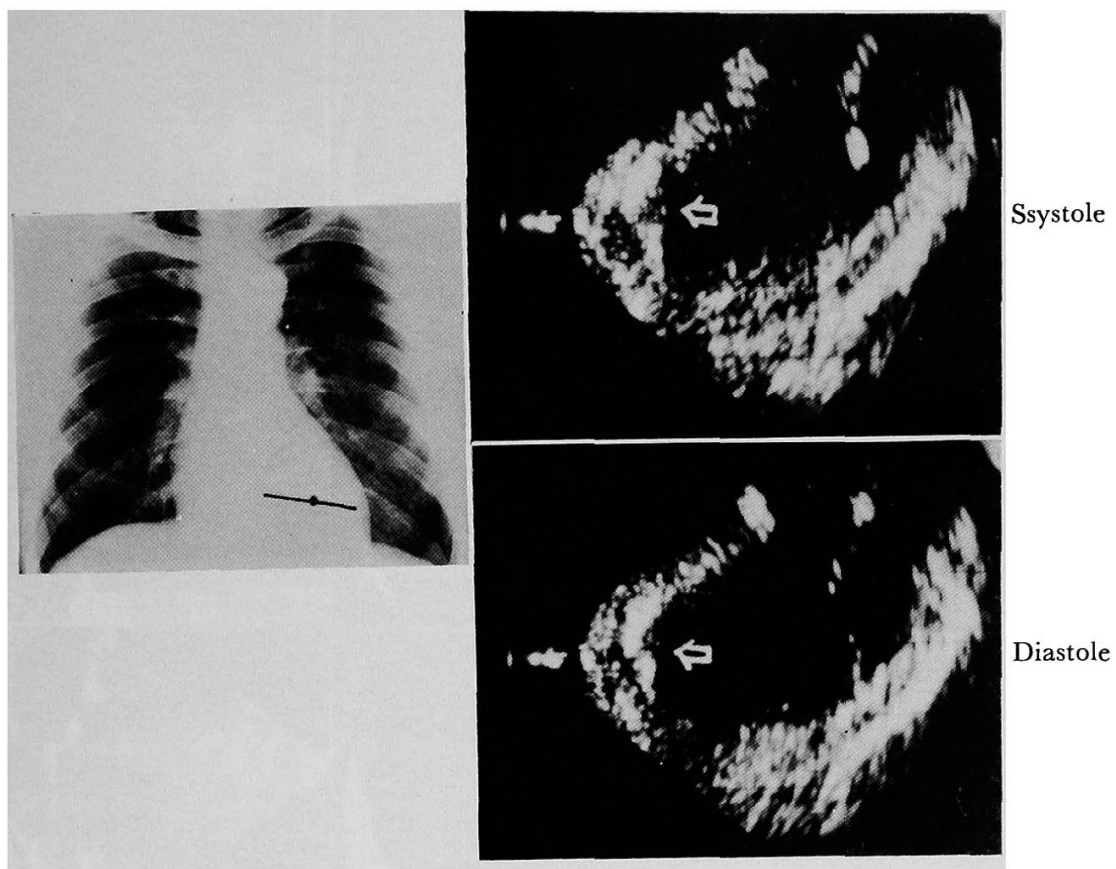

Fig. 6. Echo of antcro-lateral infarction. Infarction was diagnosed by a scan parallel to the ventricular septum with a transducer placed somewhat below and outside of the standard position. The thickness of the anterolateral wall was decreased $(\rightarrow$ arrow).

In the study of experimental myocardial infarction, the echoes related to scar were classified into 4 types. In type I, the wall echo which was thinner and more intense than that of normal myocardium had a cord-like pattern. The occurrence of this type of echo indicated the presence of a transmural scar. In type II, the thickness of the left ventricular posterior wall echo was slightly decreased. The scattered intense large nodular echoes which were observed in the infarcted area indicated the presence of transmural, scattered microscopically nodular fibrotic tissues in myocardium. When echoes of type II were found, histocytic infiltration and necrotic tissue were found around the nodular fibrotic tissue (Fig. 10).

In type III, the thickness of the left ventricular posterior wall echoes was slightly decreased, but not so markedly as that in type I.

The echo of type III showed a transmural narrow band-like pattern. This type of echo indicated that, when observed microscopically, there was a localized transmural scar in myocardium. In type IV, the thickness of the left ventricular posterior wall echo was slightly decreased and small nodular echoes were localized within the subendocardial space (Fig. 11). As shown in Figs. 10 and 11, the characteristics of abnormal echoes thus obtained were al- 


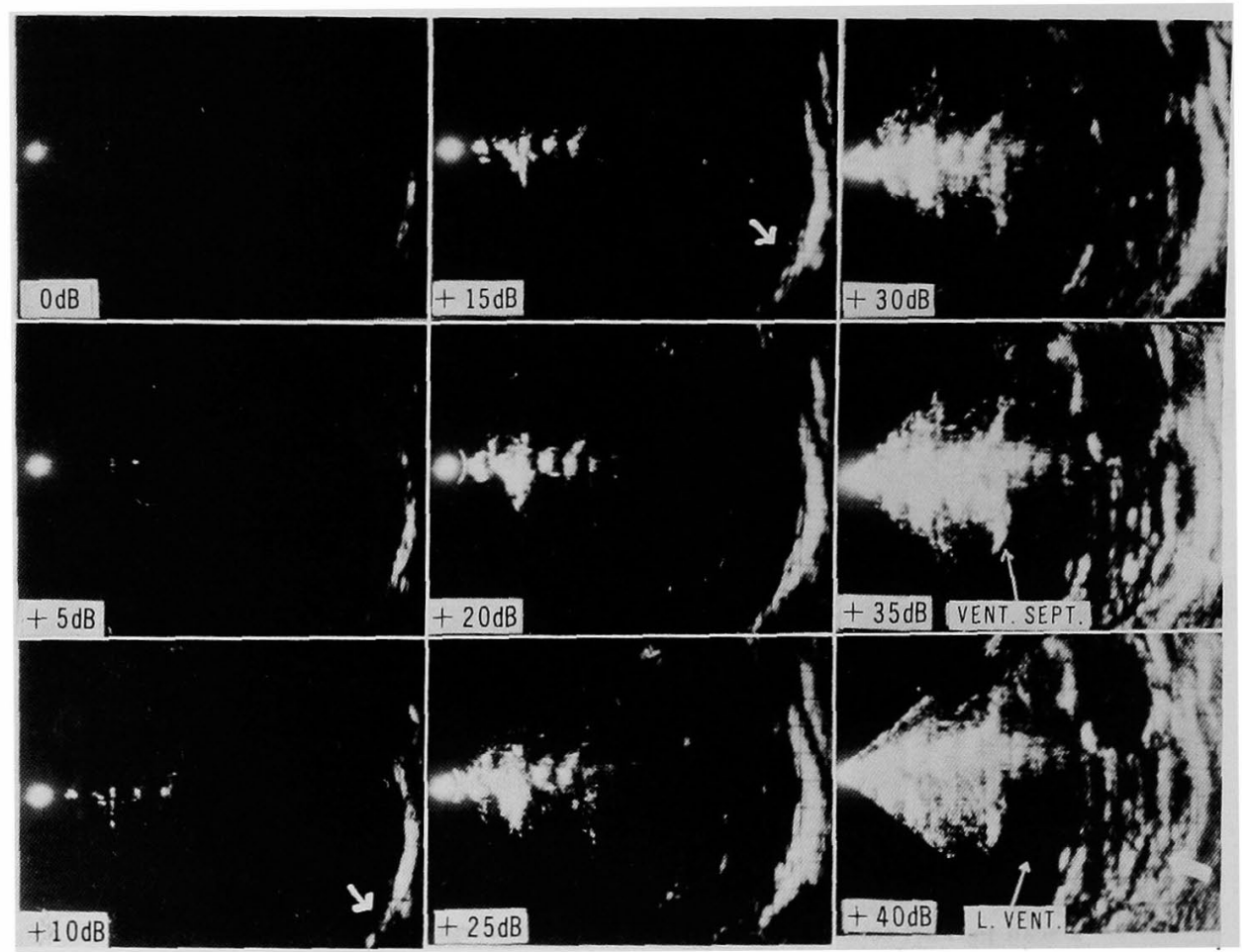

Fig. 7. Echoes of postero-inferior infarction obtained by sensitivity gradient tomographic method. Abnormal echo appears at a level of $+10 \mathrm{~dB}$ $(\rightarrow$ arrow). The echo is +10 to $+15 \mathrm{~dB}$ more intense than that of normal myocardial echo. Normal pericardial echoes are used as a reference of OdB.

most faithfully reflected in histological changes.

\section{Discussion}

Wild et $\mathrm{al}^{3)}$ using the resected human heart, reported that abnormal echoes were produced from the infarcted area. In their B-scope method, a pulsed ultrasound of extremely high frequency of $15 \mathrm{MHz}$ was utilized, so that changes could be detected only at the superficial layer of the myocardial wall. Corya and Rasmussen et $\mathbf{a l}^{\text {(4)-6) }}$ reported, based on M-mode echocardiography, that the thickness of myocardial wall echo from the infarcted area decreased and that the echo thus obtained was of a linear pattern. They reported also that the echo-intensity from the infarcted area was more intense than that of the normal myocardium. Gramiak et $\mathrm{al}^{7}$ ) demonstrated high ultrasonic reflectivity with average amplitudes 1.4-2.6 times that of normal muscle in excised dog heart imaged with a B-scanner interfaced with a computer.

When the normal myocardial tissue was damaged by obstruction of the 


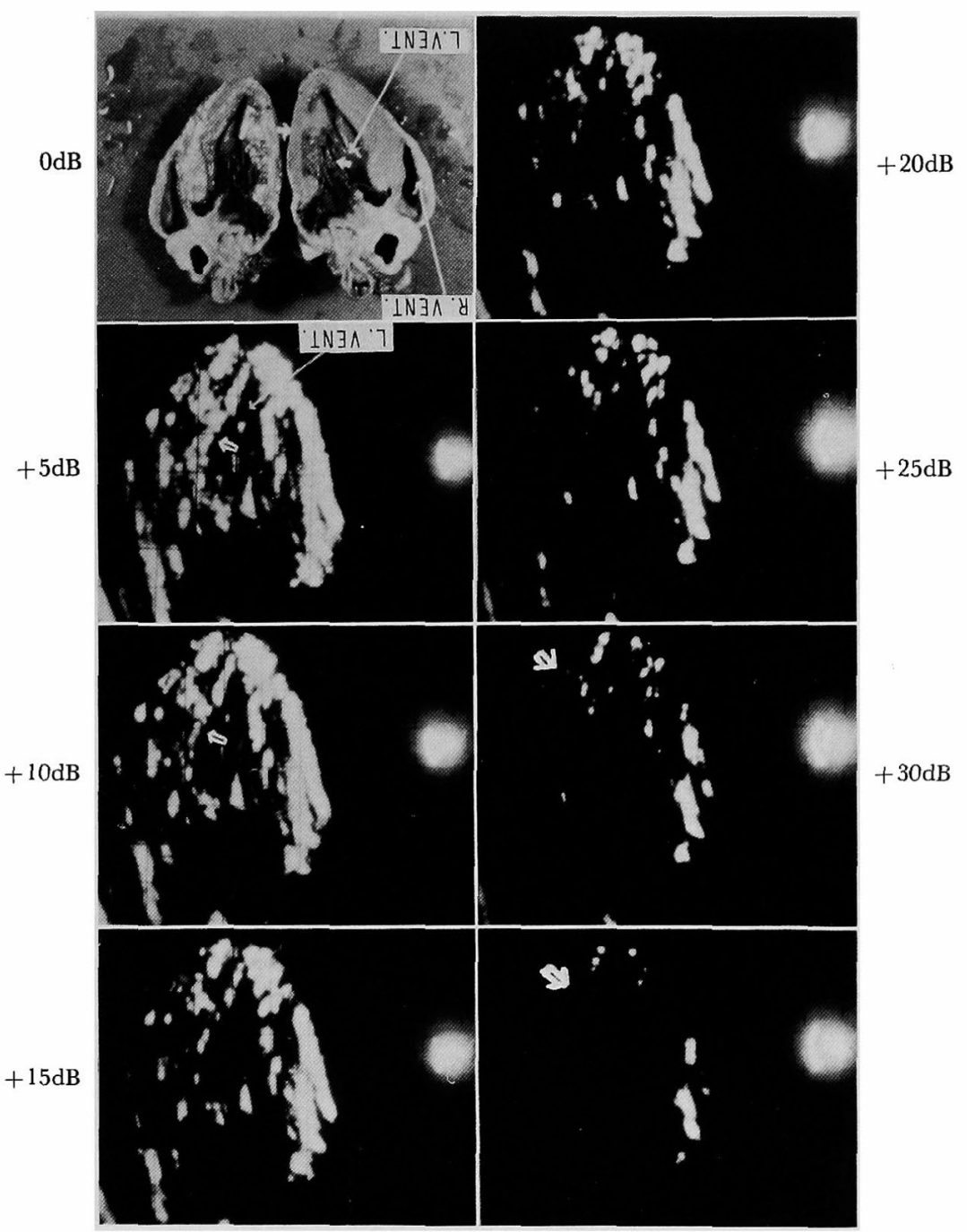

Fig. 8. Echoes of postero-inferior subendocardial infarction of an excised heart obtained by sensitivity gradient tomographic method. The abnormal echo ( $\rightarrow$ arrow) has the same intensity as that of the pericardial echo. The echo appears at a level of $0 \mathrm{~dB}$, the intensity of which is +10 to +15 $\mathrm{dB}$ more intense than that of normal myocardial echo. A postmortem specimen is shown right below. The changes in histology are almost faithfully reflected in those of the echoes.

coronary artery, the thickness of the myocardial wall echoes decreased as a result of atrophy, scar formation and the deformity of ventricular wall. The stiffness of scar results in changes of acoustic character (the changes in density of the tissue or in velocity of propagation of ultrasound through the tissue). The information thus obtained leads to the recognition of the site and extent 

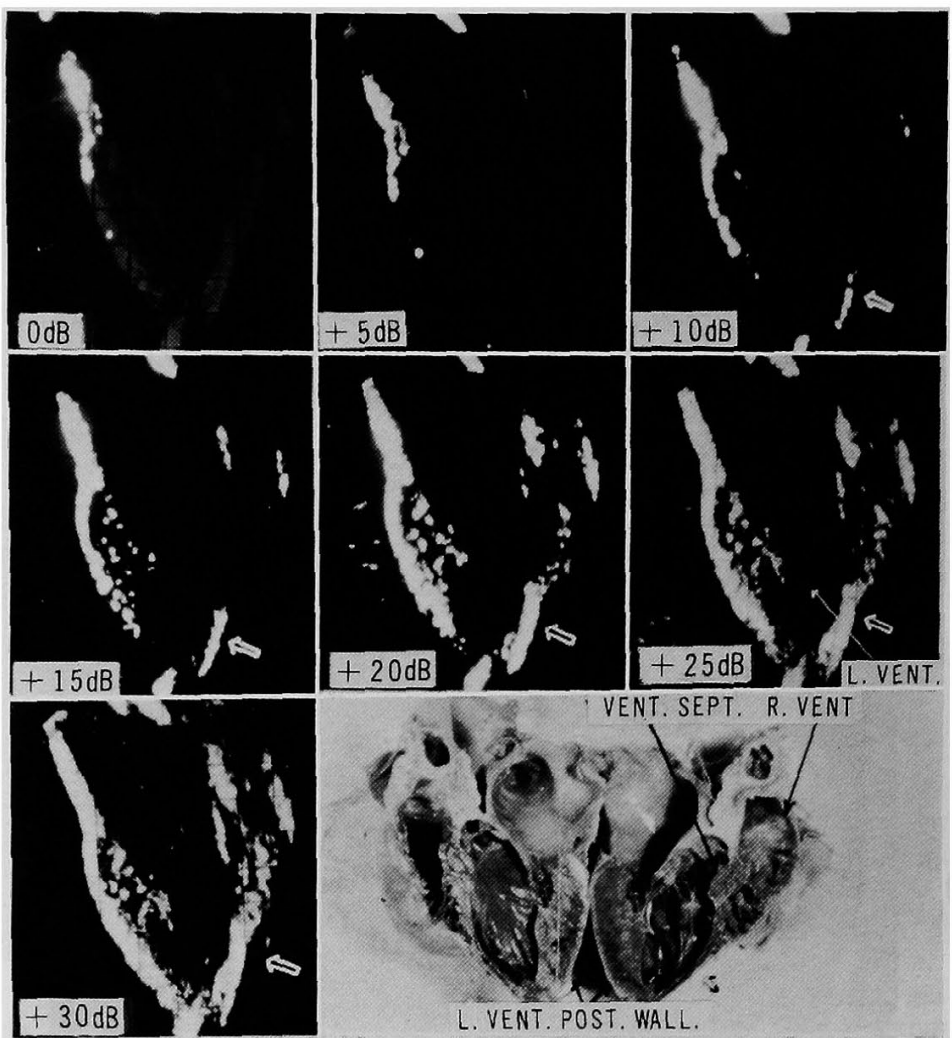

Fig. 9. Echoes of antero-septal infarction of an excised dog heat detected by sensitivity gradient tomographic method. Abnormal cord-like echo $(\rightarrow$ arrow) appears at a level of $+10 \mathrm{~dB}$. This eacho is +5 to $+10 \mathrm{~dB}$ more intense than that of the normal septal echo.

of the tissue involved in degeneration as a result of infarction.

The decrement of thickness in myocardial wall echoes was caused by the necrotic changes of myocardium and replacement with fibrotic tissue. For the collection of direct informations concerning the site and extent of such organic changes, azimuthal angle and level ultrasono-cardiotomograms obtained by changing the direction and level of ultrasonic beam along the long axis of the left ventricle are very useful.

It was found in our previous report ${ }^{81-10)}$ that echo-intensity of the tissue as well as echo pattern changed together with an increment of fibrotic tissue. Both in normal subjects and in patients with myocardial infarction, the endocardial echo is produced by a difference in acoustic impedance at the interface between the blood and the internal surface of the ventricular wall. The myocardial echo is produced by a difference in acoustic impedance at the interface between normal myocardium and the area of fibrosis. In myocardial infarc- 

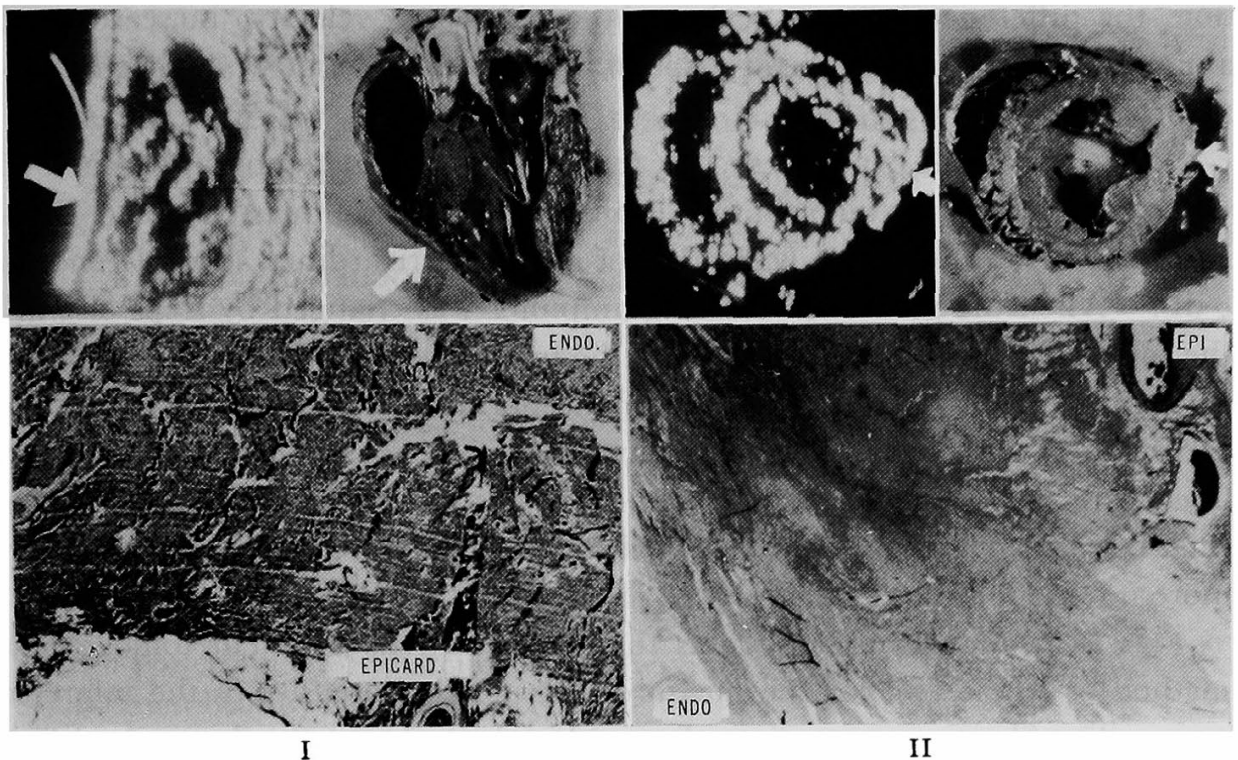

Fig, 10. Correlation between abnormal echoes on cardiotomogram and histological changes in the myocardium of experimental myocardial infarction. Type I: cord-like echo. Type II: scattered, large, nodular echoes (See text for details).

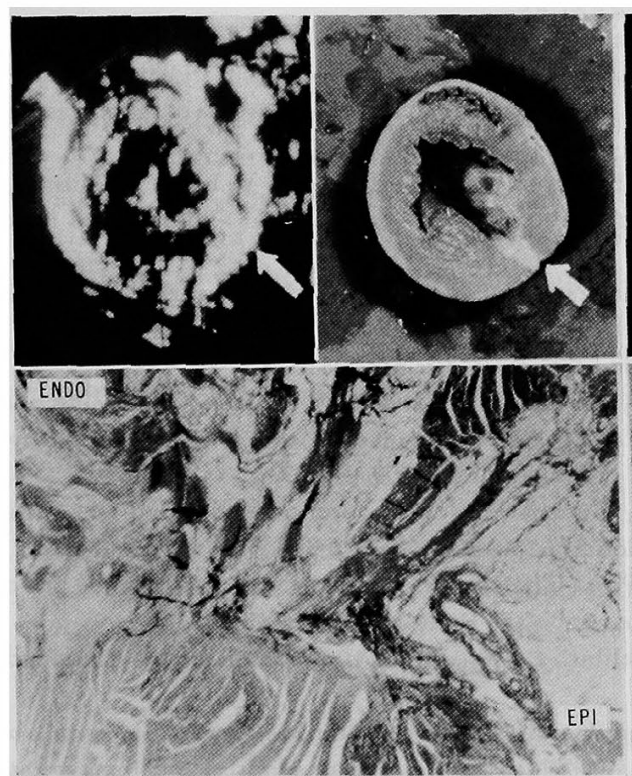

III

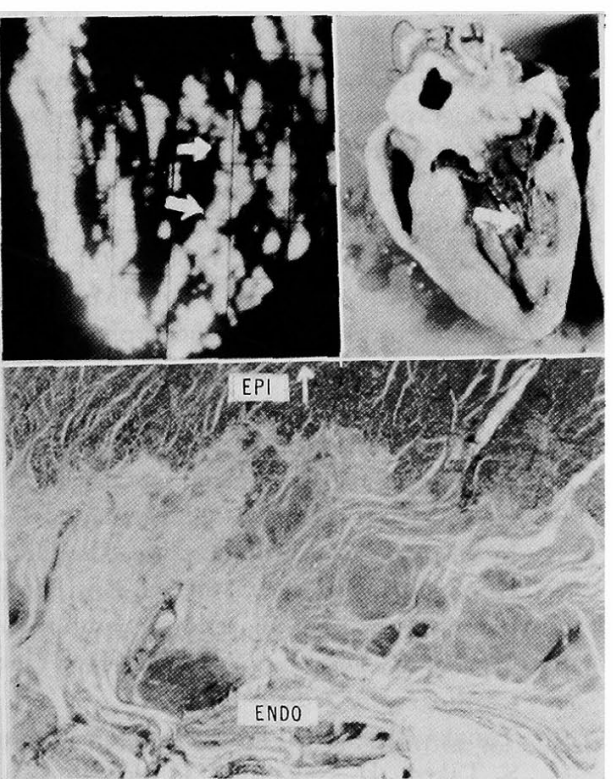

IV

Fig. 11. Correlation between abnormal echoes and histological changes of experimental myocardial infarction. Type III: a narrow band-like echo. Type IV: scattered, small, nodular echoes (See text for details). 
tion, the difference in acoustic impedance (density $\times$ velocity) increases, and abnormally strong echoes from myocardium are delineated, which are indicative of the presence of a scar.

In the present experiment, the sensitivity gradient tomographic method was applied for the quantitative evaluation of echo-intensity. The echointensity was evaluated by using that of normal pericardium as a reference of $0 \mathrm{~dB}$. When the gain of a receiving amplifier was changed by steps of $5 \mathrm{~dB}$, the intensity of abnormal echoes from the infarcted area was found to be +10 to $+15 \mathrm{~dB}$ more intense than that of the normal myocardium. Thus pericarditis and pericardial effusion associated with myocardial infarction interfered with the evaluation, when the pericardium was chosen as a reference of echo-intensity. Nevertheless, the echo from the infarcted area was always more intense than that from normal myocardium.

It is evident from these facts that the sensitivity gradient tomographic method is very useful for the measurement of echo-intensity of the infarcted area. The fibrotic myocardium showed histological changes that were different depending on the site and the range of the obstruction of coronary artery and on the degree of the development of collateral circulation. But the histological changes were found to be almost faithfully reflected in echo patterns.

\section{REFERENCES}

1. Ebina T, Oka S, Tanaka M, Terasawa Y, Unno K, Kikuchi Y, Uchida R: The ultrasonotomography for the heart and great vessels in living human subjects by means of the ultrasonic reffection technique. Jpn Heart J 8: 331, 1967

2. Tanaka M, Negazaki 'T, Kosaka S, Sugi H, Oka S, Ebina T, Terasawa Y, Unno K, Nitta K: Ultrasonic evaluation of anatomical abnormalities of heart in congenital and acquired heart disease, Brit Heart J 33:686, 1971

3. Wild JJ, Growford HD, Reid JM: Visualization of the excised human heart by means of reflected ultrasound or echography. Am Heart J 54: 903, 1957

4. Corya BC: Echocardiography in ischemic heart disease. Am J Med 63: 10, 1977

5. Corya BC, Rasmussen S, Feigenbaum H, Knoebel SB, Black MJ: Systolic thickening and thinning of the scptum and posterior wall in patients with coronary artery disease, congestive cardiomyopathy, and atrial septal defect. Circulation 55: 109, 1977

6. Rasmussen S, Corya BC, Feigenbaum H, Knoebal SB: Detection of myocardial scar tissue by M-mode echocardiography. Circulation 57: 230, 1978

7. Gramiak R, Waag RC, Schenk EA, Lee PPK, Thomson K, Macintosh P: Ultrasonic detection of myocardial infarction by amplitude analysis. Radiology $130: 713,1979$

8. Tanaka M, Terasawa Y, Hikichi H: Qualitative evaluation of the heart tissue by ultrasound. Cardiography 7: 515, 1977

9. Tanaka M, Hikichi H, Terasawa Y, Konno K, Nitta K, Kashiwagi M, Meguro T, Watanabe $S$, Takeda $\mathrm{H}$, Nakamura $\mathrm{T}$, Odagima $\mathrm{H}$ : Non-invasive detection of histological changes of the myocardium in myocardial infarction by means of ultrasonocardiotomography. Heart 11: 3, 1979

10. Tanaka M, Terasawa Y: Echocardiography: Evaluation of the tissue character in myocardium. Jpn Circulat J 43:367, 1979 